
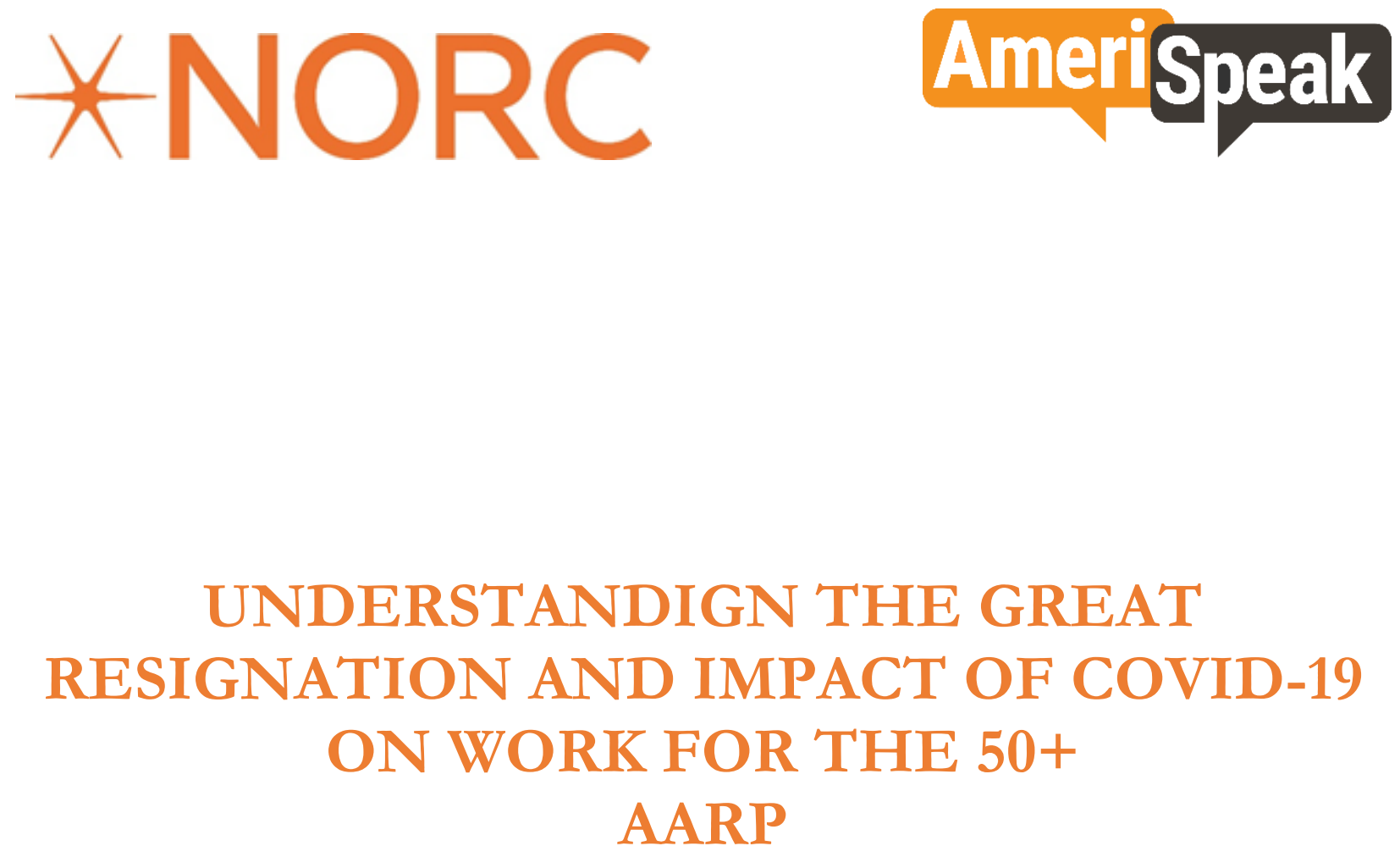

AMERISPEAK PROJECT REPOR'T

January 11, 2021

Client Contact: Rebecca Perron

NORC Account Manager:

J. Michael Dennis | Dennis-Michael@norc.org

Stefan Subias | Subias-Stefan@norc.org

NORC Project Manager:

Jacob Stolmeier | Stolmeier-Jake@,norc.org

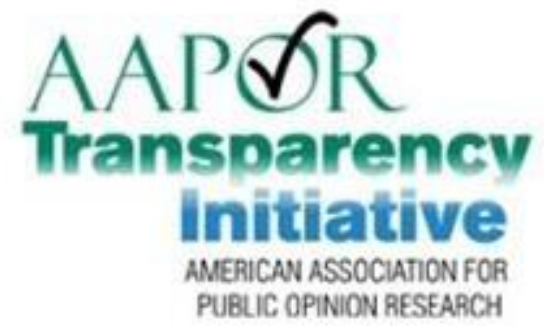

PUBLIC OPINION RESEARCH 


\section{ST'UDY INT'RODUCTION}

NORC conducted the Workforce Participation 2021 survey on behalf of Rebecca Perron at AARP using NORC's AmeriSpeak® Panel and Lucid Panel for the sample source.

The main focus of the research was to target and survey adult in U.S. population, age 50 and older, to learn more about their recent experiences in the workforce.

This study was offered in English and Spanish, in both web and phone modes.

This AmeriSpeak Project Project supplements the information provided in the NORC Card, which provides an in-depth profile of sample quality metrics for the study, the data collection field period, interview sample size, response rate statistics, the design effect, and sampling margins of error, among other statistics. Please refer to the NORC Card for information useful for compliance with the AAPOR Transparency Initiative, in addition to information provided in this AmeriSpeak Project Report.

For more detailed information on the AmeriSpeak panel recruitment and management methodology, please see the Appendix ("Technical Notes on AmeriSpeak Methodology") attached to this AmeriSpeak Project Report.

\section{SURVEY OVERVIEW}

Study Population: General Population Age 50+

Sample Units: 8792

Completed Units: 2910

Expected Eligibility Rate: 100\%

Observed Eligibility Rate: 100\%

Margin of Error: \pm 2.65 percentage points (pp)

Avg. Design Effect: 2.33

Survey Field Period: December 14, 2021 - December 27, 2021

Median Duration (minutes): 3

Definitions of the Above Categories:

Study Population: The total set of individuals of interest to which the researcher intends to apply their conclusions.

Sample Units: The number of panel members selected into the study sample.

Completed Units: The number of sample units that completed the interview based on the study-specific definition of what constitutes a complete interview. This number excludes any cases removed due to data quality concerns.

Expected Eligibility Rate: The percentage of the sampling population who are expected to meet study eligibility criteria.

Observed Eligibility Rate: The percentage of the sample members who were eligible for the study among those who answered the screening questions.

Design Effect: The design effect is the variance under the complex design divided by the variance under an SRS (simple random sampling) design of the same sample size. The reported design effect is an approximation based on the coefficient of variation of the final survey weight.

Margin of Error: Margin of error is defined as half the width of the $95 \%$ confidence interval for a proportion estimate of $50 \%$ adjusted for design effect. It is therefore the largest margin of error possible for all estimated percentages based on the study sample.

Survey Field Length: the period from the earliest to the latest contact dates of cases sampled for the survey.

Duration: Length of time for completed interviews. Interview length is calculated differently depending upon whether the interview was conducted over the phone or via the web. For telephone mode, it is the time 
from when the respondent picks up the telephone until they hang up the telephone. For web interviews, it is the time from when they first connect to the web system to the time they log off the system or become inactive. In the case of multiple contacts, this number represents the sum of those contacts.

\section{STUDY-SPECIFIC DETAILS}

\section{Sampling}

A general population sample of U.S. adults age 50 and older was selected from NORC's AmeriSpeak Panel for this study, with oversamples to reach at least 600 Hispanics and 600 African American respondents.

The sample for a specific study is selected from the AmeriSpeak Panel using sampling strata based on age, race/Hispanic ethnicity, education, and gender (24 sampling strata in total). The size of the selected sample per sampling stratum is determined by the population distribution for each stratum. In addition, sample selection takes into account expected differential survey completion rates by demographic groups so that the set of panel members with a completed interview for a study is a representative sample of the target population. If panel household has one more than one active adult panel member, only one adult in the household is eligible for selection (random within-household sampling). Panelists selected for an AmeriSpeak study earlier in the business week are not eligible for sample selection until the following business week.

For technical information about the AmeriSpeak Panel, including recruitment process and panel management policies, please see the Appendix.

The AmeriSpeak panel sample was supplemented with respondents from the Lucid nonprobability online opt-in panel to achieve the Latino oversample.

\section{Distribution of Completed Interviews in Delivered Analysis File \\ by Sample Source and Race/Ethnicity (Unweighted)}

\begin{tabular}{|l|c|c|c|c|c|c|}
\hline \multicolumn{1}{|c|}{ Demographic } & \multicolumn{2}{c|}{ Lucid Sample Source } & NORC AmeriSpeak Source & \multicolumn{2}{c|}{ Total } \\
\hline & N Interviews & $\%$ & N Interviews & $\%$ & N Interviews & $\%$ \\
\hline Latino/as & 775 & $100 \%$ & 539 & $18.5 \%$ & 1314 & $35.7 \%$ \\
\hline $\begin{array}{l}\text { White, Non-Hispanic } \\
\text { (incl. multi-White) }\end{array}$ & 0 & $0 \%$ & 1657 & $56.9 \%$ & 1657 & $45.0 \%$ \\
\hline $\begin{array}{l}\text { African Americans, Non- } \\
\text { Hispanic } \\
\text { (incl. multi-Black) }\end{array}$ & 0 & $0 \%$ & 612 & $21.0 \%$ & 612 & $16.6 \%$ \\
\hline All Other, Non-Hispanic & 0 & $0 \%$ & 102 & $3.5 \%$ & 102 & $2.8 \%$ \\
\hline Total & 775 & $100 \%$ & 2910 & $100.0 \%$ & 3685 & $100 \%$ \\
\hline
\end{tabular}

\section{Field}

A sub-sample AmeriSpeak web-mode panelists were invited to the survey on December 14, 2021 in a softlaunch. The initial data from the soft-launch was reviewed and the remainder of sampled AmeriSpeak panelists were invited to the survey on December 15, 2021.

In total NORC collected 3,685 interviews, 3,420 by web mode and 265 by phone mode.

\section{Panel \& Survey Sample Performance}

To meet AAPOR Transparency Initiative, we offer performance outcome measures of both the AmeriSpeak Panel and the sample. The information in this section applies only to the AmeriSpeak 
probability portions of the sample. These kinds of measures have no meaning with non-probability sample from Lucid. The AmeriSpeak Panel is a household panel, so recruitment and retention rates are household rates. The survey sample is an individual-level sample pulled from the AmeriSpeak panel, so those are individual-level rates.

\begin{tabular}{|c|c|}
\hline \multicolumn{2}{|c|}{ Panel Outcome Measures } \\
\hline $\begin{array}{c}\text { Weighted Household } \\
\text { Panel Recruitment Rate } \\
\text { (WPRecr) }\end{array}$ & $\begin{array}{c}\text { Weighted Household Panel } \\
\text { Retention Rate } \\
\text { (WPRet) }\end{array}$ \\
\hline $17.1 \%$ & $75.6 \%$ \\
\hline
\end{tabular}

Weighted Household (HH) Recruitment Rate_WPRecr): The weighted AAPOR RR III for the AmeriSpeak panel recruitment corresponding to the recruitment cohorts sampled for the study. A recruited household is a household where at least one adult successfully completed the recruitment survey and joined the panel.

Weighted Household (HH) Retention Rate (WPRet): The weighted percent of recruited households that are still available for sampling for this survey among the recruitment cohorts sampled for the study.

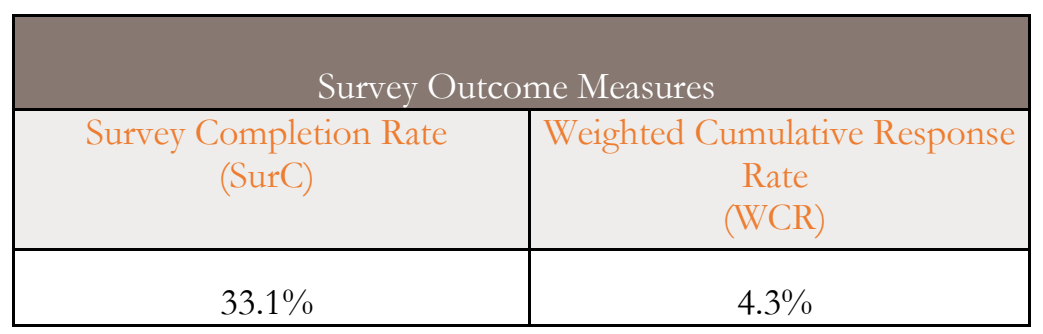

Survey Completion Rate (SurC): The percent of sample members who completed the survey interview of those who were invited to take it. 8792 panelists were invited to the survey and 2910 completed the survey.

Weighted Cumulative Response Rate (WCR): The overall survey response rate that accounts for the survey completion rate, plus it includes panel outcome measures such as panel recruitment rate and panel retention rate. This overall rate is weighted to account for the sample design and differential inclusion probabilities of sample members. $(W C R=S u r C \times W P R e t \times W P R e c r)$

\section{Gaining Cooperation of AmeriSpeak Panelists for the Study}

To encourage study cooperation, NORC sent email reminders to sampled web-mode panelists on the following dates:

- December 18, 2021

- December 21, 2021

- December 23, 2021

- December 24, 2021

- December 26, 2021

- December 27, 2021

To administer the phone-survey, NORC dialed the sampled phone-mode panelists throughout the field period. 
Panelists were offered the cash equivalent of $\$ 2$ for completing this survey.

Data processing

NORC prepared a fully labeled data file of respondent survey data and demographic data.

NORC applied cleaning rules to the survey data for quality control by removing survey responses in the main study interview questions from non-eligible respondents. These respondents either took the survey in less than one-third the median time to complete or skipped more than twenty percent of eligible questions.

\section{Statistical Weighting}

Statistical weights for the study eligible respondents were calculated using panel base sampling weights to start.

Panel base sampling weights for all sampled housing units are computed as the inverse of probability of selection from the NORC National Frame (the sampling frame that is used to sample housing units for AmeriSpeak) or address-based sample. The sample design and recruitment protocol for the AmeriSpeak Panel involves subsampling of initial non-respondent housing units. These subsampled non-respondent housing units are selected for an in-person follow-up. The subsample of housing units that are selected for the nonresponse follow-up (NRFU) have their panel base sampling weights inflated by the inverse of the subsampling rate. The base sampling weights are further adjusted to account for unknown eligibility and nonresponse among eligible housing units. The household-level nonresponse adjusted weights are then post-stratified to external counts for number of households obtained from the Current Population Survey. Then, these household-level post-stratified weights are assigned to each eligible adult in every recruited household. Furthermore, a person-level nonresponse adjustment accounts for nonresponding adults within a recruited household.

Finally, panel weights are raked to external population totals associated with age, sex, education, race/Hispanic ethnicity, housing tenure, telephone status, and Census Division. The external population totals are obtained from the Current Population Survey. The weights adjusted to the external population totals are the final panel weights.

\section{Panel Weighting Variables \& the Variable Categories}

Age: 18-24, 25-29, 20-39, 40-49, 50-59, 60-64, and 65+

Gender: Male and Female

Census Division: New England, Middle Atlantic, East North Central, West North Central, South Atlantic, East South Central, West South Central, Mountain, and Pacific

Race/Ethnicity: Non-Hispanic White, Non-Hispanic Black, Hispanic, and Non-Hispanic Other

Education: Less than High School, High School/GED, Some College, and BA and Above

Housing Tenure: Home Owner and Other

Household phone status: Cell Phone-only, Dual User, and Landline-only/Phoneless

Age x Gender: 18-34 Male, 18-34 Female, 35-49 Male, 35-49 Female, 50-64 Male, 50-64 Female, 65+ Male, and 65+ Female

Age x Race/Ethnicity: 18-34 Non-Hispanic White, 18-34 All Other, 35-49 Non-Hispanic White, 3549 All Other, 50-64 All Other, 50-64 All Other, 65+ Non-Hispanic White, and 65+ All Other

Study-specific base sampling weights are derived using a combination of the final panel weight and the probability of selection associated with the sampled panel member. Since not all sampled panel members respond to the survey interview, an adjustment is needed to account for and adjust for survey non-respondents. This adjustment decreases potential nonresponse bias associated with sampled panel members who did not complete the survey interview for the study. Thus, the nonresponse adjusted survey weights for the study are adjusted via a raking ratio method to target population totals associated with the following topline sociodemographic characteristics: age, sex, education, race/Hispanic ethnicity, and Census region, and the following socio-demographic interactions: age x gender, age x AARP membership. Additionally, as we 
oversample African-American and Hispanic respondents, our raking ratio method takes into account the interaction of the topline demographic characteristics and respondent race/Hispanic ethnicity.

\section{Study-Specific Post-Stratification Weighting Variables \& the Variable Categories}

RAC3 x Age: 50-59 Non-Hispanic Black, 60-64 Non-Hispanic Black, and 65+ Non-Hispanic Black, 18-24 Hispanic, 25-29 Hispanic, 30-39 Hispanic, 40-49 Hispanic, 50-59 Hispanic, 60-64 Hispanic, and $65+$ Hispanic

RAC3 x Gender: Non-Hispanic Other Male and Non-Hispanic Other Female, Non-Hispanic Black Male and Non-Hispanic Black Female, Hispanic Male and Hispanic Female

RAC3 x Census Region: Non-Hispanic Other Northeast, Non-Hispanic Other Midwest, NonHispanic Other South, and Non-Hispanic Other West, Non-Hispanic Black Northeast, NonHispanic Black Midwest, Non-Hispanic Black South, and Non-Hispanic Black West, Hispanic Northeast, Hispanic Midwest, Hispanic South, and Hispanic West

RAC3 x Education: Non-Hispanic Other Less than High School, Non-Hispanic Other High School/GED, Non-Hispanic Other Some College, and Non-Hispanic Other BA and Above, Non-Hispanic Black Less than High School, Non-Hispanic Black High School/GED, NonHispanic Black Some College, and Non-Hispanic Black BA and Above, Hispanic Less than High School, Hispanic High School/GED, Hispanic Some College, and Hispanic BA and Above

Race/Ethnicity: Non-Hispanic White, Non-Hispanic Black, Hispanic, and Non-Hispanic All Other Age x Gender: 50-64 Male, 50-64 Female, 65+ Male, and 65+ Female

Age x AARP Membership: 50-64 Member, 50-64 Non-member, 65+ Member, 65+ Non-member

The weights adjusted to the external population totals are the final AmeriSpeak sample weights.

Raking and re-raking is done during the weighting process such that the weighted demographic distribution of the survey completes resemble the demographic distribution in the target population. The assumption is that the key survey items are related to the demographics. Therefore, by aligning the survey respondent demographics with the target population, the key survey items should also be in closer alignment with the target population.

TrueNorth Calibration for Nonprobability Sample - To incorporate the nonprobability sample, NORC used TrueNorth calibration services, an innovative hybrid calibration approach developed at NORC based on small area estimation methods to explicitly account for potential bias associated with the nonprobability sample ${ }^{12}$. The purpose of TrueNorth calibration is to adjust the weights for the nonprobability sample to bring weighted distributions of the nonprobability sample in line with the population distribution for characteristics correlated with the survey variables. Such calibration adjustments help to reduce potential bias, yielding more accurate population estimates.

First, the nonprobability sample is raked for each main race/ethnicity group to the following sociodemographic characteristics: age, sex, education, and Census Region, and to the socio-demographic interactions: age $\mathrm{x}$ sex, age $\mathrm{x}$ AARP membership.

Second, the weighted AmeriSpeak sample and the calibrated nonprobability sample were used to develop a small area model to support domain-level estimates, where the domains were defined by race/ethnicity, age,

${ }^{1}$ Ganesh, N., Pineau, V., Chakraborty, A., Dennis, J.M., (2017). "Combining Probability and Non-Probability Samples Using Small Area Estimation.” Joint Statistical Meetings 2017 Proceedings.

${ }^{2}$ Yang, Y. Michael, Nada Ganesh, Ed Mulrow, and Vicki Pineau. (2018). "Estimation Methods for Nonprobability Samples with a Companion Probability Sample," Proceedings of the Joint Statistical Meetings, 2018. 
and gender. The dependent variables for the models were key survey variables. The model included covariates, domain-level random effects, and sampling errors. The covariates were external data available from other national surveys such as health insurance, internet access, voting behavior, and housing type from the American Community Survey (ACS) or the Current Population Survey (CPS).

Finally, the combined AmeriSpeak and nonprobability sample weights were derived such that for the combined sample, the weighted estimate reproduced the small domain estimates (derived using the small area model) for key survey variables.

Design Effect and Sampling Margin of Error Calculations

Study design effect: 2.33

Study margin of error: $+/-2.65 \%$

Margin of error for NH All Other: +/- 3.23\%

Margin of error for NH Black (includes multi-race Black): $+/-5.76 \%$

Margin of error for Latino/Hispanic: +/- 4.67\%

Under TrueNorth calibration, which applies to the Hispanics/Latino oversample, combined probability and nonprobability sample yields approximately unbiased estimates. The margins of error reported here reflect the sampling variation of the probability sample as well as the TrueNorth model-assisted calibration procedures that generate the combined sample weights. As such, it is reasonable for analysts using this data to employ standard methods for approximating margins of error and statistical significance, although there is no statistically agreed upon approach to doing this when utilizing nonprobability samples. 


\section{Benchmark Comparisons}

The following table shows the weighted and unweighted results for key demographics and compares them to population benchmarks. The fourth column shows the percentage point difference between population benchmarks and the final weighted results.

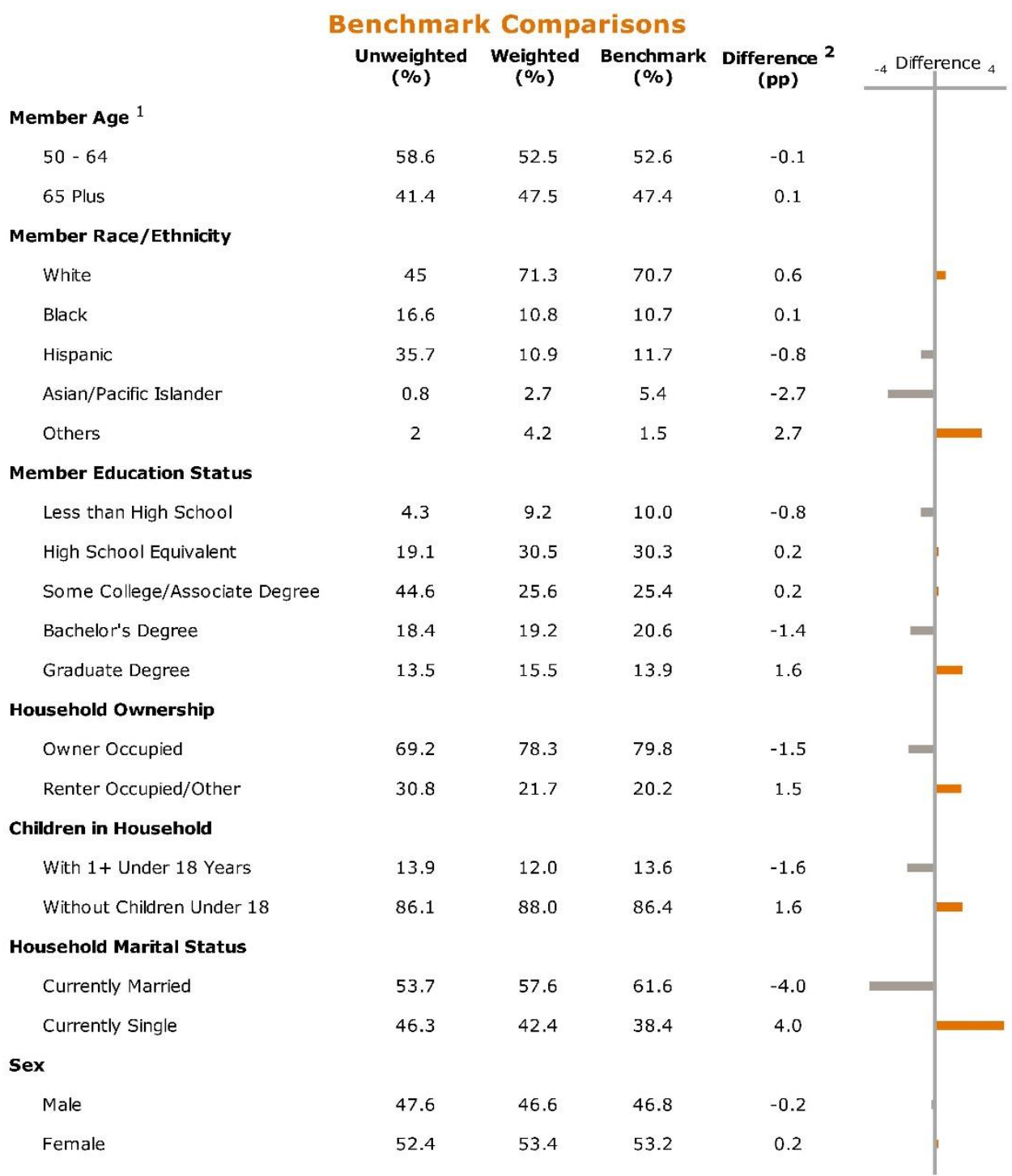

Response rates are calculated based on the probability sample. MOF, deff, and benchmark comparisons are based on combined probability and non-probability sanuples.

${ }^{1}$ Race/Ethnicity, Ilousehold Ownership. Income, Education, Number of Children, Marital Status, Gender, and Geographic Region benchnzarks are from the March 2021 Census Bureau Current Population Survey. ${ }^{2}$ The difference between the Weighted and Benchmark colurms.

As a part of the AAPOR Transparency Initiative, it is incumbent on us to state that there are no perfect studies, and all research and methods have their limitations. The purpose of this document is to make apparent, for this study, some possible limitations, the steps taken to minimize them, and the potential or known sources of measurable or estimated error whenever possible. However, there is always going to be some unmeasured and unknowable error with all forms of public opinion research, including ours. 


\section{Deliverables}

The following files were created for Rebecca Perron as part of the study deliverables:

- Survey interview data file in SPSS format

- Annotated Questionnaire

- Codebook in Excel format

- Final questionnaire - programming format, in Word document

- Final questionnaire - simple format, in Word document

- Project report documenting study procedures

- NORC Card

- Banner tables in Excel formats

\section{HOW TO DESCRIBE AMERISPEAK AND NORC @ THE UNIVERSITY OF CHICAGO}

For purposes of publication, when describing AmeriSpeak and its panel methodology, we recommend using the following language:

Funded and operated by NORC at the University of Chicago, AmeriSpeak ${ }^{\circledR}$ is a probability-based panel designed to be representative of the US household population. Randomly selected US households are sampled using area probability and address-based sampling, with a known, nonzero probability of selection from the NORC National Sample Frame. These sampled households are then contacted by US mail, telephone, and field interviewers (face to face). The panel provides sample coverage of approximately $97 \%$ of the U.S. household population. Those excluded from the sample include people with P.O. Box only addresses, some addresses not listed in the USPS Delivery Sequence File, and some newly constructed dwellings. While most AmeriSpeak households participate in surveys by web, non-internet households can participate in AmeriSpeak surveys by telephone. Households without conventional internet access but having web access via smartphones are allowed to participate in AmeriSpeak surveys by web. AmeriSpeak panelists participate in NORC studies or studies conducted by NORC on behalf of governmental agencies, academic researchers, and media and commercial organizations.

For more information, email AmeriSpeak-BD@norc.org or visit AmeriSpeak.norc.org.

If editors or reviewers are requesting anything more specific or any other detail, please reach out to us to make certain you are using accurate language.

NORC at the University of Chicago is best described as follows:

NORC at the University of Chicago is an independent research institution that delivers reliable data and rigorous analysis to guide critical programmatic, business, and policy decisions. Since 1941, NORC has conducted groundbreaking studies, created and applied innovative methods and tools, and advanced principles of scientific integrity and collaboration. Today, government, corporate, and nonprofit clients around the world partner with NORC to transform increasingly complex information into useful knowledge. Please visit www.norc.org for more information. 


\title{
APPENDIX
}

\section{TECHNICAL OVERVIEW OF THE AMERISPEAK® PANEL NORC'S PROBABILITY-BASED HOUSEHOLD PANEL}

\author{
Updated June 16, 2020
}

This technical overview provides the basic information about AmeriSpeak ${ }^{\circledR}$, a large probability-based panel funded and operated by NORC at the University of Chicago. AmeriSpeak is designed to be representative of the U.S. household population, including all 50 states and the District of Columbia. U.S. households are randomly selected with a known, non-zero probability from the NORC National Frame as well as addressbased sample (ABS) frames, and then recruited by mail, telephone, and by field interviewers face to face. AmeriSpeak panelists participate in NORC studies or studies conducted by NORC on behalf of governmental agencies, academic institutions, the media, and commercial organizations.

The construction of the AmeriSpeak panel started in 2014 with pilot samples. In 2015, about 7,000 households were recruited from a sample of around 60,000 addresses. In 2016, about 128,000 addresses were sampled to expand the panel to around 20,000 recruited households. About 51,000 addresses were selected for the 2017 recruitment, which led to the expansion of the regular AmeriSpeak panel to 23,000 recruited households. The AmeriSpeak Panel expanded to approximately 30,000 households in 2018 and 35,000 households in 2019 through further recruitment efforts. The 2020 recruitment is expected to expand the panel by another 5,000 households. The current panel size is 48,900 panel members age 13 and over residing in over 40,000 households.

In addition to the regular panel for general population studies, AmeriSpeak also contains sub-panels to support studies of special populations, including AmeriSpeak Latino, AmeriSpeak Teen, and AmeriSpeak Young Adult 18-34 (which features an oversample of African Americans, Hispanics, and Asians). AmeriSpeak is also the probability sample source for TrueNorth $^{\circledR}$, the NORC calibration solution for combining probability and non-probability samples for estimation through small area modeling that leverages data from AmeriSpeak, the American Community Survey, Current Population Survey, and other data sources for improved statistical efficiency. ${ }^{3}$

\section{Panel Sample Frame}

The primary sampling frame for AmeriSpeak is the 2010 NORC National Frame, a multistage probability sample that fully represents the U.S. household population. We provide a brief description of how the National Frame was constructed after the 2010 Census.

The primary sampling units (PSUs) in the first stage sample selection are 1,917 National Frame Areas (NFAs), each of which is an entire metropolitan area (made up of one or more counties), a county, or a group of counties with a minimum population of 10,000. A total of 126 NFAs are selected in the first stage, including 38 certainty NAFs, 60 urban NFAs, and 28 non-urban NFAs. The largest 38 NFAs, those with a population of at least 1,543,728 ( 0.5 percent of the 2010 Census U.S. population), were selected into the National Frame with certainty. The certainty NFAs consist of areas with high population density and are dominated by census tracts with city-style mailing addresses. These areas contain 56 percent of the U.S. household population while only accounting for about 8 percent of the geographic area of the United States. The remaining 1,879 NFAs are stratified into urban areas where city-style addresses predominate, and rural areas that are less likely to have city-style addresses. The latter stratum comprises 81 percent of the geographic area, but only 14 percent of the population. A sample of 60 and 28 NFAs is selected systematically

\footnotetext{
${ }^{3}$ For more information about TrueNorth, see http://amerispeak.norc.org/our-capabilities/Pages/TrueNorth.aspx.
} 
from the urban and rural stratum, respectively, with selection probability proportional to size (PPS) where the measure of size is the number of housing units per NFA.

Within the 126 selected NFAs, the secondary sampling units (SSUs) are segments defined from Census tracts or block groups, where each segment contains at least 300 housing units according to the 2010 Census.

Within the certainty NFAs, a sample of 896 segments was selected using systematic PPS sampling, where the size of a segment is the number of housing units. Implicit stratification was achieved by sorting the segments by location (NFA, state, and county), by principal city indicator, by ethnic and income indicators. From each urban and rural NFA, a sample of 8 and 5 segments was selected, respectively, using systematic PPS sampling where the measure of size is the number of housing units per segment. A total of 618 segments are selected from the non-certainty NFAs ${ }^{4}$. Overall, a stratified probability sample of 1,514 segments was selected into the National Frame in the second stage sampling.

Within the selected segments, all housing units are listed using the U.S. Postal Service Delivery Sequence File (DSF). In the 123 segments where the DSF coverage is deemed inadequate, the DSF address list is enhanced with in-person field listing to improve coverage. The final National Frame, consisting of all listed households in the sample segments, is estimated to provide over 97 percent coverage of the U.S. household population. It contains almost 3 million households, including over 80,000 rural households that are added through inperson listing. In addition to NORC's National Frame, the DSF is used as a supplemental sample frame in four states. Although nationally representative, the National Frame does not include households from Alaska, Iowa, North Dakota, and Wyoming. Since 2016, the annual panel recruitment sample has included a small address-based sample (ABS) from these four states to assure AmeriSpeak presence in in all U.S. States and Washington, D.C.

In 2017, an enhanced DSF frame was also used to develop a new Latino Panel with adequate representation of Spanish-language-dominant Hispanics. Census tracts with high incidence (at least 30\%) of Spanishdominant Hispanics were targeted for this recruitment. Furthermore, within these Census tracts, households that were flagged as Hispanic based on consumer vendor data (that are typically used for direct-mail marketing) were oversampled. This new AmeriSpeak Latino Panel contains approximately 5,400 Hispanic panelists with $24 \%$ of those panelists being Spanish-language dominant. As of February $2020,63.4 \%$ of the recruited adults in the AmeriSpeak Panel were sourced from the National Frame, 27.1\% from targeted ABS frames, and $9.5 \%$ from voter registration files ${ }^{5}$. Proper weights allow the full use of the combined sample.

\section{Panel Sample Selection}

For panel sample selection between 2014 and 2018, National Frame segments are stratified into six sampling strata based on the race/ethnicity and age composition of each segment, as below:

- Hispanic, high youth segments

- Hispanic, not high youth segments

- Non-Hispanic Black, high youth segments

- Non-Hispanic Black, not high youth segments

- Other, high youth segments

- Other, not high youth segments

Hispanic segments are those where Hispanics make up at least a third of the population and the Hispanic share in the population is greater than that of non-Hispanic Black. Similarly, non-Hispanic Black segments are

\footnotetext{
${ }^{4}$ A sample of 5 segments was selected from each of the 28 non-urban NFAs. However, 2 sample segments were later subsampled out in Montana due to cost.

${ }^{5}$ Voter registration files were used as supplemental sample source for the AmeriSpeak Young Adult Panel.
} 
those where non-Hispanic Black make up at least a third of the population and the non-Hispanic Black share in the population is greater than that of Hispanic. Finally, High Youth refers to segments in which 18-24 year old adults are at least $12 \%$ of the total adult population. The above stratification is used to oversample housing units in areas with higher concentration of young adults, Hispanics, and non-Hispanic AfricanAmericans. The resulting household sample is referred to as the initial AmeriSpeak sample or sample for initial panel recruitment.

To support the second stage of panel recruitment, initially sampled but nonresponding housing units are subsampled for a nonresponse follow-up (NRFU) ${ }^{6}$. At this stage, consumer vendor data are matched to the pending housing units, and housing units that are flagged as having a young adult ${ }^{7}$ (18-34 years of age) or minority (Hispanic ${ }^{8}$, non-Hispanic Black ${ }^{9}$ ) are oversampled for the NRFU sample. Overall, approximately one in five initially nonresponding housing units are subsampled for NRFU using the same six sampling strata defined above. Due to NRFU, these initially nonresponding housing units have a much higher selection probability compared to the housing units that were recruited during the first stage of panel recruitment.

A 2-phase state-based ABS sample design was used for the 2019 AmeriSpeak recruitment. NORC's National Frame is designed to represent the U.S. household population nationally. At the state level, however, the panel may have more significant clustering effects from the use of the National Frame, especially for states with a small population. The primary objective of the 2019 design is to improve state-level representation by selecting the recruitment sample mostly from areas that are outside the National Frame. A stratified systematic sample was selected in the first phase, where each state constitutes a sampling stratum and the sample was allocated to the strata proportional to the square root of the state population. In the second phase, young adults, Hispanic, non-Hispanic Black, and conservatives are oversampled based on commercial data sources to improve their representation in the panel. Because the 2019 design did not use NRFU face-toface recruitment, the 2019 design did not involve geographic clustering.

The overall AmeriSpeak Panel sample design reflects the cumulative design features of the NORC National Frame and the annual recruitment samples thus far. These design features are captured in the final panel weight for each recruited household and each panelist. There are mainly two reasons why the sampling design for AmeriSpeak Panel recruitment deviates from EPSEM (Equal Probability of Selection Method) sampling: (a) oversampling of housing units in segments with a higher concentration of young adults and minorities results in the sample selection probabilities being higher for housing units in these segments; and (b) NRFU subsampling results in initially nonresponding housing units having a much higher selection probability. Furthermore, within the NRFU samples, selection probabilities vary for housing units depending on the appended commercial data flags to target specific demographics for improved efficiency. The initial and NRFU sampling procedures are examined and possibly modified each year to more efficiently recruit subpopulations who are less likely to respond to the recruitment survey.

\section{Panel Recruitment Procedures}

The highly efficient recruitment procedures set AmeriSpeak apart from other national panels. AmeriSpeak Panel recruitment is a two-stage process: (i) an initial recruitment using USPS mailings, telephone contact, and

\footnotetext{
${ }^{6}$ A small fraction of initially nonresponding housing units are not eligible for NRFU, including "hard refusals" and those with an appointment for a call back from NORC.

${ }^{7}$ A young adult flagged household refers to a household where MSG or TargetSmart indicated there was an 18-24 year old adult in the household. In 2016 and 2017, a slightly different definition was used, and a young adult flagged household was defined as having an 18-34 year old adult in the household by MSG or 18-30 year old adult by TargetSmart.

${ }^{8}$ A Hispanic flagged household refers to a household where MSG or TargetSmart indicated the presence of a Hispanic adult in the household.

${ }^{9}$ A non-Hispanic Black flagged household refers to a household where MSG or TargetSmart indicated the presence of a non-Hispanic Black adult in the household.
} 
modest incentives, and (ii) a more elaborate NRFU recruitment using FedEx mailings, enhanced incentives, and in-person visits by NORC field interviewers.

For the initial recruitment, sample households are invited to join AmeriSpeak online by visiting the panel website AmeriSpeak.org or by calling a toll-free telephone line (in-bound/outbound supported). Both English and Spanish languages are supported for online and telephone recruitment. The initial recruitment data collection protocol features the following: an over-sized pre-notification postcard, a USPS recruitment package in a 9"x12" envelope (containing a cover letter, a summary of the privacy policy, FAQs, and a study brochure), two follow-up post cards, and contact by NORC's telephone research center for sample units with a matched telephone number.

For the second-stage NRFU recruitment, a stratified random sample is selected from the nonrespondents of the initial recruitment. Units sampled for NRFU are sent a new recruitment package by Federal Express with an enhanced incentive offer. Meanwhile, NORC field interviewers make personal, face-to-face visits to the pending cases to encourage participation. Once the households are located, the field interviewers administer the recruitment survey in-person using CAPI or else encourage the respondents to register online or by telephone.

\section{Panel Recruitment Response Rate and Other Panel Statistics}

A sample household is considered recruited or responded if at least one adult in the household joins the panel. The weighted household response rate (AAPOR RR3) is about $6 \%$ for initial recruitment and $28 \%$ for NRFU recruitment. We report two recruitment response rates: one for all the panel recruitment years (20142019) and one for the recruitment years with NRFU (2014-2018). For all recruitment years, the cumulative weighted household response rate is $24.1 \%$; for recruitment years with NRFU, and the cumulative weighted household response rate is $34.2 \%$. For client studies requiring a panel recruitment response rate exceeding $30 \%$, the sampling frame may be restricted to the panelists recruited in the NRFU years. The panel recruitment response rate calculation methodology is consistent with AAPOR guidelines and fully documented. ${ }^{10}$ The annual panel retention rate is about $85 \%$.

For individual client surveys based on the AmeriSpeak Panel, the AAPOR RR3 response rate is between 10\% to $20 \%$ depending on specific study parameters such as target population, survey length, time in the field, salience of subject, and the like. This response rate takes into account panel recruitment rate, panel retention rate, and survey participation rate. ${ }^{11}$

Other important panel statistics with respect to the 2014-2019 recruited households are as follows: 62\% are recruited in the initial stage and 38\% are recruited via NRFU; $85 \%$ of the active panelists prefer to do web or online surveys, while $15 \%$ prefer to participate in telephone surveys; $21 \%$ of the recruited households are

\footnotetext{
${ }_{10}$ See http://amerispeak.norc.org/research/Pages/WhitePaper_ResponseRateCalculation_AmeriSpeak_2016.pdf

${ }_{11}$ A properly calculated cumulative AAPOR response rate for panel-based research takes into account all sources of non-response at each stage of the panel recruitment, management, and survey administration process (see https://www.aapor.org/AAPOR Main/media/publications/Standard-Definitions20169theditionfinal.pdf, page 48-9). A common misapplication of the term "response rate" in online panel surveys is to represent the survey-specific cooperation rate as the "cumulative survey response rate." See "Response Rate Calculation Methodology for Recruitment of a Two-Phase Probability-Based Panel: The Case of AmeriSpeak” authored by Robert Montgomery, J. Michael Dennis, N. Ganesh. The paper is available at https://amerispeak.norc.org/research/.
} 
non-Internet ${ }^{12} ; 82 \%$ are cell-phone only or cell-phone mostly; 19\% are African-American and 20\% Hispanic; and $33 \%$ have household income below $\$ 30,000$ (compared to CPS benchmark of $26 \%$ ). ${ }^{13}$

\section{Impact of Non-Response Follow-Up}

NRFU is instrumental in producing the industry-leading response rate for AmeriSpeak Panel recruitment. Moreover, due to the more intensive effort, NRFU recruitments better represent hard to reach groups and are therefore more representative of the target population. For example, initial recruitment tends to underrepresent young adults 18-34 years of age. NRFU recruitment corrects for this bias by bringing the age distribution of the panel closer to ACS benchmarks.

Overall, NRFU recruitment significantly improves the representation of the panel with respect to demographic segments that are under-represented among the respondents to the initial recruitment, including young adults (persons 18 to 34 years of age), African Americans, Hispanics, lower income households, renters, cell-phone only households, and persons with lower educational attainment (e.g., no college degree). To the extent that these demographic characteristics are correlated with substantive survey variables, NRFU helps to reduce potential non-response bias in the sample estimates. NORC's research indicates that NRFU respondents are indeed somewhat different from initial respondents for many common survey variables. For example, compared to the panelists recruited during the initial stage, NRFU panelists tend to be more conservative politically, more likely to attend church, less interested in current events or topics in the news report, less knowledgeable about science, less likely to be in favor of gun control policies, less likely to read a print newspaper (more likely to read the news online and use social media), more likely to eat at fast food restaurants and so on ${ }^{14}$. These observations illustrate that NRFU recruitment is critical for achieving a more balanced panel and for making the substantive estimates in AmeriSpeak studies more accurate. Even though NRFU panelists are more reluctant to complete surveys, the addition of NRFU panelists reduced total absolute bias on average 5 to21 percentage points when compared to the initial stage recruits (among examined surveys). ${ }^{15}$

\section{Mixed-Mode Data Collection}

The AmeriSpeak Panel supports mixed-mode data collection to improve response rate and the representativeness of the complete surveys. During the recruitment survey, AmeriSpeak panelists are offered an opportunity to choose their preferred mode-web or phone-for future participation in AmeriSpeak surveys. A recruited household can consist of both web- and phone-mode panelists residing in the same household. Panelists predominantly prefer web over phone mode. As of February 2020, 85\% of the active panelists prefer to do web or online surveys, while 15\% prefer to participate in telephone surveys. The telephone mode encompasses panelists without internet access, panelists whose only internet access is via a smartphone, and panelists with internet access but are unwilling to share an email address.

\footnotetext{
12 The non-internet households (HHs) are those that do not select "High-speed, broadband internet at home (such as cable or DSL)" or "Dial-up internet at home" response options when they are asked "What kind of internet access do you have? Please select all that apply" item in the recruitment survey. The non-internet HHs include those that only use internet on a cell connection or mobile phone.

${ }^{13}$ For transparency purposes, unweighted percentages are presented in this section. Hence, these results do not take into account selection probabilities. The base weighted distributions that take into account selection probabilities can be provided upon request.

14 See "The Undercounted: Measuring the Impact of 'Nonresponse Follow-up' on Research Data and Outcome Measures" authored by Ipek Bilgen, J. Michael Dennis, N. Ganesh. The paper will be soon available at https://amerispeak.norc.org/research/.

15 See "Nonresponse Follow-up Impact on AmeriSpeak Panel Sample Composition and Representativeness" authored by Ipek Bilgen, J. Michael Dennis, N. Ganesh. The paper is available at https://amerispeak.norc.org/research/.
} 
To the extent that non-internet households or "net averse" persons are different from the rest of the population, mixed-mode surveys have better population coverage and produce more accurate population estimates. NORC's telephone interviewers administer the telephone surveys using a data collection system supporting both the phone and web modes, providing an integrated sample management and data collection platform. For panelists using smartphones for web-mode surveys, the NORC survey system renders an optimized presentation of the survey questions for these mobile users.

Panel Management and Maintenance

Panel management and maintenance are crucial for panel health and efficiency. NORC maintains strict panel management rules to limit respondent burden, reduce panel attrition, and minimize the risk of panel fatigue. On average, AmeriSpeak panelists are invited to participate in client studies two to three times a month. AmeriSpeak works with NORC clients to create surveys that provide an appropriate user experience for AmeriSpeak panelists. AmeriSpeak will not field surveys that in our professional judgment will result in a poor user experience for our panelists. AmeriSpeak also has a designated website and a telephone number for panelist communications.

Panel maintenance is a dynamic process because the AmeriSpeak Panel is supplemented and refreshed regularly over time to grow the panel, compensate for panel attrition, and improve panel representation for specific subpopulations. For example, the Latino Panel and Teen Panel are created to support studies of Hispanics and teenagers, respectively; the 2019 recruitment is primarily designed to improve sample representation at the state level. As panelists are added or/and removed from the panel, the panel refreshment process takes place to ensure that the refreshed panel fully represents the corresponding target population.

\section{ABOUT NORC AT THE UNIVERSIT'Y OF CHICAGO}

As one of the world's foremost independent research institutions, NORC at the University of Chicago delivers objective data and meaningful analysis to help decision-makers and leading organizations make informed choices and identify new opportunities. Since 1941, NORC has applied sophisticated methods and tools, innovative and cost-effective solutions, and the highest standards of scientific integrity and quality to conduct and advance research on critical issues. Today, NORC expands on this tradition by partnering with government, business, and nonprofit clients to create deep insight across a broad range of topics and to disseminate useful knowledge throughout society.

Headquartered in downtown Chicago, NORC works in over 40 countries around the world, with additional offices on the University of Chicago campus, the DC metro area, Atlanta, Boston, and San Francisco.

\section{ADDITIONAL RESOURCES}

To learn more about AmeriSpeak or to share an RFP, please contact AmeriSpeak at AmeriSpeakBD@,norc.org. Information about AmeriSpeak capabilities and research papers are available online at AmeriSpeak.NORC.org. 